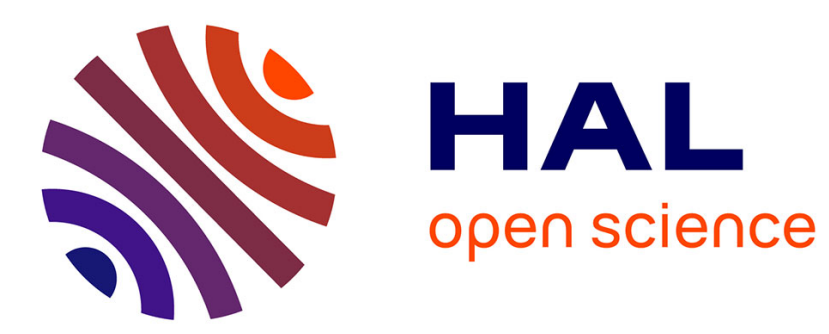

\title{
On-line simultaneous maximization of the size and the weight for degradable intervals schedules
}

Fabien Baille, Evripidis Bampis, Christian Laforest, Nicolas Thibault

\section{To cite this version:}

Fabien Baille, Evripidis Bampis, Christian Laforest, Nicolas Thibault. On-line simultaneous maximization of the size and the weight for degradable intervals schedules. COCOON 2005, 2005, China. pp.308-317, 10.1007/11533719_32. hal-00341366

\section{HAL Id: hal-00341366 https://hal.science/hal-00341366}

Submitted on 18 Jul 2009

HAL is a multi-disciplinary open access archive for the deposit and dissemination of scientific research documents, whether they are published or not. The documents may come from teaching and research institutions in France or abroad, or from public or private research centers.
L'archive ouverte pluridisciplinaire HAL, est destinée au dépôt et à la diffusion de documents scientifiques de niveau recherche, publiés ou non, émanant des établissements d'enseignement et de recherche français ou étrangers, des laboratoires publics ou privés. 


\title{
On-line simultaneous maximization of the size and the weight for degradable intervals schedules
}

\author{
Fabien Baille, Evripidis Bampis, Christian Laforest, Nicolas Thibault
}

Tour Evry 2, LaMI, Université d'Evry, 523 place des terrasses, 91000 EVRY France

\{fbaille, bampis, laforest, nthibaul\}@lami.univ-evry.fr

\begin{abstract}
We consider the problem of scheduling on-line a sequence of degradable intervals in a set of $k$ identical machines. Our objective is to find a schedule that maximizes simultaneously the Weight (equal to the sum of processing times) and the Size (equal to the number) of the scheduled intervals. We propose a bicriteria algorithm that uses the strategies of two monocriteria algorithms (GOL [7], maximizing the Size and LR [4], maximizing the Weight) and yields two simultaneous constant competitive ratios. This work is an extension of [2] (COCOON'04), where the same model of degradable intervals was investigated in an off-line context and the two objectives were considered separately.
\end{abstract}

In this paper, we consider the problem of scheduling on-line degradable intervals on $k$ identical machines. We define a degradable interval $\sigma$ by a triplet $(r, q, d)$ where $r$ denotes the release date, $q$ the minimal deadline and $d$ the deadline $(r<q \leq d)$. This means that $\sigma$ is scheduled if and only if it is executed from date $r$ to date $t(q \leq t \leq d)$ on one machine. Intuitively, in this model, each interval can be shortened (with respect to the required total execution $[r, d)$ ). We denote by $[r, t)$ the numerical interval corresponding to the effective execution of a degradable interval $\sigma$ and by $p(\sigma)=t-r$ its processing time. We define the weight $w(\sigma)$ of the effective execution of any interval $\sigma$ by $w(\sigma)=t_{\sigma}-r_{\sigma}$. This means that the weight of an interval $\sigma$ is equal to its processing time (it is known in the literature as the proportional weight model [8]). In our model, we consider on-line sequences of degradable intervals $\sigma_{1}, \ldots, \sigma_{n}$ where the $\sigma_{i}$ 's are revealed one by one in the increasing order of their release dates $\left(r_{1} \leq r_{2} \leq \cdots \leq r_{n}\right)$, and future intervals are not known in advance.

For any algorithm $A$, we denote by $A^{k}$ the version of $A$ running on $k$ identical machines. In our model, an on-line algorithm $A^{k}$ has to build at each step a valid schedule. A schedule is valid if and only if for every date $t$, there is at most one interval on each machine and each interval is scheduled at most once. When a new interval $\sigma_{i}$ is revealed (at step $i$ ), the algorithm $A^{k}$ can reject it (in this case, it is definitively lost) or serve it. In this second case, if the algorithm schedules $\sigma_{i}$ on machine $j$, it interrupts at least the already scheduled interval intersecting $\sigma_{i}$ on machine $j$. The interrupted intervals are definitively lost and no gain is obtained from them for both metrics. Thus, each step $i$ of any on-line algorithm $A^{k}$ can be decomposed into two stages: First, there is the interrupting stage of step $i$. During this stage, the algorithm interrupts a subset of the already 
scheduled intervals (note that this subset can be empty). Secondly, there is the scheduling stage of step $i$. During this stage, the algorithm decides if the new interval $\sigma_{i}$ is served or rejected, and if it is served, on which machine $\sigma_{i}$ is served.

Notation 1 (Schedule $A^{k}\left(\sigma_{1}, \ldots, \sigma_{i}\right)$ ) Let $\sigma_{1}, \ldots, \sigma_{n}$ be any on-line sequence of degradable intervals and let $A^{k}$ be any algorithm running on $k$ identical machines. For every step $i(1 \leq i \leq n)$, we denote by $A^{k}\left(\sigma_{1}, \ldots, \sigma_{i}\right)$ the schedule returned by $A^{k}$ at the end of step $i$.

We define the size $N(O)=|\{\sigma \in O\}|$ (i.e. the number of scheduled intervals) and the weight $W(O)=\sum_{\sigma \in O} w(\sigma)$ (i.e. the weight of scheduled intervals) of any schedule $O$. Our problem is then to find a schedule which has size and weight the largest possible. In order to evaluate the quality of a schedule for our measures (the Size and the Weight), we use the competitive ratio [5].

Definition 1 (Competitive ratio). Let $\sigma_{1}, \ldots, \sigma_{n}$ be any on-line sequence of intervals. Let $A^{k}\left(\sigma_{1}, \ldots, \sigma_{i}\right)$ be the schedule on $k$ machines given by an algorithm $A^{k}$ at step $i(1 \leq i \leq n)$ and let $O_{i}^{*}$ be the optimal (off-line) schedule on $k$ machines of $\left\{\sigma_{1}, \ldots, \bar{\sigma}_{i}\right\}$ for the criterion $C$ (here, $C=W$ or $\left.C=N\right)$. $A^{k}$ has a competitive ratio of $\rho$ (it is $\rho$-competitive) for the criterion $C$ if and only if we have:

$$
\forall i, 1 \leq i \leq n, \rho \cdots C\left(A^{k}\left(\sigma_{1}, \ldots, \sigma_{i}\right)\right) \geq C\left(O_{i}^{*}\right)
$$

An algorithm $A^{k}$ is $(\rho, \mu)$-competitive if it is simultaneously $\rho$-competitive for the Size and $\mu$-competitive for the Weight. In this paper, we propose a $\left(\frac{k}{r}, \frac{4 k}{k-r-2}\right)$ competitive algorithm, called $A B^{k}$ (with $1 \leq r<k$ ). For example, if we set $r=\frac{k}{2}$ (if $\frac{k}{2} \geq 3$ and $k$ even), $A B^{k}$ is $\left(2, \frac{8}{1-\frac{4}{k}}\right)$-competitive.

Previous works. The off-line version of the bicriteria non-degradable problem has been treated in [3] where a $\left(\frac{k}{r}, \frac{k}{k-r}\right)$-approximation algorithm $(1 \leq r<k)$ has been proposed. Concerning the monocriteria non-degradable problems, they have been extensively studied for both the off-line and the on-line versions. In particular, the off-line versions are polynomial (see Faigle and Nawijn [7] for the Size and Carlisle and Lloyd [6] or Arkin and Silverberg [1] for the Weight problems). In the on-line context, the algorithm GOL of Faigle and Nawijn [7] is optimal for the Size problem. For the Weight problem, there is a series of works going from the paper of Woeginger [8] to the paper of Bar-Noy et al. [4], who proposed on-line algorithms with constant competitive ratios. Note that the two degradable monocriterion intervals problem has been investigated in [2].

Outline of the paper. In Section 1, we present two monocriterion algorithms $\left(G O L^{k}[7]\right.$ and $\left.L R^{k}[4]\right)$ in the degradable interval model. Section 2 is devoted to the description and the analysis of our on-line bicriteria algorithm $A B^{k}$ using $G O L$ and $L R$ as subroutines.

\section{Two on-line monocriteria algorithms for the size and the proportional weight metrics}

In this section, we describe and analyze the competitiveness of the algorithm of Faigle and Nawijn [7] and the algorithm of Bar-Noy et al. [4]. We use them as 
subroutines of our algorithm $A B^{k}$ (see Section 2) in order to obtain a pair of constant competitive ratios.

The algorithm $G O L^{k}$. We describe the algorithm $G O L^{k}$ of [7] in the degradable interval model by decomposing it into an interrupting stage and a scheduling stage.

Algorithm $G O L^{k}$ (adaptation of [7])

When a new interval $\sigma_{i}$, defined by $\left(r_{i}, q_{i}, d_{i}\right)$, is revealed, choose its effective execution $\sigma_{i}^{q}=\left[r_{i}, q_{i}\right)$ (i.e. each new interval is totally degraded) and do:

Interrupting stage: If there are $k$ served intervals intersecting the date $r_{i}$, let $\sigma_{\max }$ be the one with the maximum deadline.

If $\sigma_{\max }$ does not exist (there is a free machine), do not interrupt any interval. Else, If $d_{\max } \geq q_{i}$ then interrupt $\sigma_{\max }$.

If $d_{\max }<q_{i}$ then do not interrupt any interval.

Scheduling stage:

If there is a free machine, then schedule $\sigma_{i}^{q}$ on it.

Else, reject $\sigma_{i}$.

Note that the original algorithm of [7] is described for the classical non-degradable interval model. In the following, we denote by $G O L_{N}^{k}$ this original version of the algorithm (notice that it is the same algorithm as $G O L^{k}$, except that $G O L_{N}^{k}$ does not degrade any interval).

Lemma $1 G^{k} L^{k}$ is optimal for the Size in the degradable interval model.

Proof. Let $\sigma_{1}, \ldots, \sigma_{n}$ be an on-line sequence of intervals such that for all $i$, $1 \leq i \leq n, \sigma_{i}$ is defined by $\left(r_{i}, q_{i}, d_{i}\right)$. Let $\sigma_{1}^{D}, \ldots, \sigma_{n}^{D}$ be the sequence such that for all $i, 1 \leq i \leq n, \sigma_{i}^{D}=\left[r_{i}, q_{i}\right)$ (i.e. the sequence version with the intervals totally degraded). Let $O_{D}^{*}$ be an optimal schedule of $\sigma_{1}, \ldots, \sigma_{n}$ in the degradable interval model and let $O_{N}^{*}$ be an optimal schedule of $\sigma_{1}^{D}, \ldots, \sigma_{n}^{D}$ in the nondegradable interval model. By [2], we know that $N\left(O_{D}^{*}\right)=N\left(O_{N}^{*}\right)$. Furthermore, since $G O L_{N}^{k}$ is optimal for the Size in the non-degradable interval model (See $[7])$, we have $N\left(O_{N}^{*}\right)=N\left(G O L_{N}^{k}\left(\sigma_{1}^{D}, \ldots, \sigma_{n}^{D}\right)\right)$. By definition of $G O L^{k}$ we have $G O L_{N}^{k}\left(\sigma_{1}^{D}, \ldots, \sigma_{n}^{D}\right)=G O L^{k}\left(\sigma_{1}, \ldots, \sigma_{n}\right)$. If we combine all these equalities, we obtain $G O L^{k}\left(\sigma_{1}, \ldots, \sigma_{n}\right)=G O L_{N}^{k}\left(\sigma_{1}^{D}, \ldots, \sigma_{n}^{D}\right)=N\left(O_{N}^{*}\right)=N\left(O_{D}^{*}\right)$. Thus, $G O L^{k}$ is optimal for the Size in the degradable interval model.

The algorithm $L R^{k}$. We now describe the algorithm $L R^{k}$ of [4] adapted to our degradable interval model and decomposed into an interrupting stage and a scheduling stage (for all $k \geq 3$ ).

\section{Algorithm $L R^{k}$ (adaptation of [4])}

We denote by $F_{t}$ the set of scheduled intervals containing date $t$. When a new interval $\sigma_{i}$ defined by $\left(r_{i}, q_{i}, d_{i}\right)$ is revealed, choose the effective execution $\sigma_{i}^{d}=$ $\left[r_{i}, d_{i}\right)$ (i.e. do not degrade any interval) and do:

Interrupting stage:

If $\left|F_{r_{i}}\right|<k$, then do not interrupt any interval.

If $\left|F_{r_{i}}\right|=k$, then 
1. Sort the $k+1$ intervals of $F_{r_{i}} \cup\left\{\sigma_{i}^{d}\right\}$ by increasing order of release dates. If several intervals have the same release date, order them in the decreasing order of their deadlines and let $L$ be the set of the $\left\lceil\frac{k}{2}\right\rceil$ first intervals.

2. Sort the $k+1$ intervals of $F_{r_{i}} \cup\left\{\sigma_{i}^{d}\right\}$ by decreasing order of deadlines (ties are broken arbitrarily) and let $R$ be the set of the $\left\lfloor\frac{k}{2}\right\rfloor$ first intervals.

If $\sigma_{i}^{d} \in L \cup R$, then interrupt any interval $\sigma_{j}$ in $F_{r_{i}}-L \cup R$,

Else do not interrupt any interval.

Scheduling stage:

If $\left|F_{r_{i}}\right|<k$, then schedule $\sigma_{i}^{d}$ on any free machine.

If $\left|F_{r_{i}}\right|=k$ and $\sigma_{i}^{d} \in L \cup R$, then schedule $\sigma_{i}^{d}$ on the machine where $\sigma_{j}$ has been interrupted.

If $\left|F_{r_{i}}\right|=k$ and $\sigma_{i}^{d} \notin L \cup R$, then reject $\sigma_{i}$.

In the following, we show that $L R^{k}$ is $\left(\frac{4}{1-\frac{2}{k}}\right)$-competitive in the degradable interval model for the Weight metric (note that this is very close to 4 when $k$ is large). We first show that the weight of an optimal degradable schedule is no more than twice the weight of an optimal non-degradable schedule.

Lemma 2 For every set of intervals $\left\{\sigma_{1}, \ldots, \sigma_{n}\right\}$, let $O^{* n d}$ be an optimal schedule of $\left\{\sigma_{1}, \ldots, \sigma_{n}\right\}$ for the proportional weight metric in the non-degradable interval model (i.e. $q_{i}=d_{i}$ ), and let $O^{* d}$ be an optimal schedule of $\left\{\sigma_{1}, \ldots, \sigma_{n}\right\}$ for the same metric in the degradable interval model. We have:

$$
W\left(O^{* d}\right) \leq 2 W\left(O^{* n d}\right)
$$

Proof. Let $O_{1}^{* d}, \cdots, O_{k}^{* d}$ be the $k$ sub-schedules of $O^{* d}\left(O_{i}^{* d}\right.$ executes the same intervals at the same dates as machine $i$ of $\left.O^{* d}\right)$. Thus, we have:

$$
W\left(O^{* d}\right)=\sum_{i=0}^{k} W\left(O_{i}^{*}\right)
$$

Let $\Gamma_{i}=\left\{\sigma_{j} \in\left\{\sigma_{1}, \ldots, \sigma_{n}\right\}: \sigma_{j} \in O_{i}^{* d}\right\} . O_{i}^{* d}$ is an optimal schedule of $\Gamma_{i}$ in the degradable interval model. Indeed, suppose, by contradiction, that there exists a valid schedule $O$ of $\Gamma_{i}$ such that $W\left(O_{i}^{* d}\right)<W(O)$. This means that the valid schedule consisting in the union of the $O_{j}^{* d}$ 's, except for $j=i$, which is replaced by $O$, generates a weight greater than $O^{* d}$ and is valid. This contradicts the optimality of $O^{* d}$.

Let us apply the 2-approximation algorithm for one machine schedules described in [2] separately on each $\Gamma_{i}(1 \leq i \leq k)$. Let $O_{1}, \cdots, O_{k}$ be the obtained schedules. Thus, by Theorem 5 of [2], for each $i$, we have $W\left(O_{i}^{* d}\right) \leq 2 W\left(O_{i}\right)$. We sum the $k$ inequalities and we obtain $W\left(O^{* d}\right) \leq 2 \sum_{i=1}^{k} W\left(O_{i}\right)$. Moreover, since the 2-approximation algorithm of [2] does not degrade the intervals, the $k$ machine schedule consisting in the union of the $O_{i}$ 's is valid for the non-degradable interval model. This means that $\sum_{i=1}^{k} W\left(O_{i}\right) \leq W\left(O^{* n d}\right)$. Combining this last inequality with $W\left(O^{* d}\right) \leq 2 \sum_{i=1}^{k} W\left(O_{i}\right)$ leads to:

$$
W\left(O^{* d}\right) \leq 2 W\left(O^{* n d}\right)
$$

Corollary $1 L R^{k}$ is $\left(\frac{4}{1-\frac{2}{k}}\right)$-competitive for the degradable interval model on $k \geq 3$ machines. 
Proof. It is known that $L R^{k}$ is $\left(\frac{2}{1-\frac{2}{k}}\right)$-competitive for the non-degradable interval model (from an adaptation of the proof of [4]). Thus, by definition, we have $\frac{2}{1-\frac{2}{k}} W\left(L R^{k}(\sigma)\right) \geq W\left(O^{* n d}\right)$. By Lemma 2 , we have $2 W\left(O^{* n d}\right) \geq W\left(O^{* d}\right)$. Combining these two inequalities leads to $\frac{4}{1-\frac{2}{k}} W\left(L R^{k}(\sigma)\right) \geq W\left(O^{* d}\right)$. This means that $L R^{k}$ is $\left(\frac{4}{1-\frac{2}{k}}\right)$-competitive for the degradable model.

\section{Our algorithm $A B^{k}$}

Definition 2 (Cover relation). Let $\sigma$ be an interval defined by the triplet $(r, q, d)$. Let $\sigma^{1}=\left[r, t_{1}\right)$ and $\sigma^{2}=\left[r, t_{2}\right)$ be two valid effective executions of $\sigma$ (i.e. $q \leq t_{1} \leq d$ and $q \leq t_{2} \leq d$ ). We say that $\sigma^{1}$ covers $\sigma^{2}$ if and only if $\sigma^{2} \subseteq \sigma^{1}$ (i.e. if and only if $t_{2} \leq t_{1}$ ).

Definition 3 (Union $^{+}$of sets of degraded intervals). Let $\left\{\sigma_{1}, \cdots, \sigma_{n}\right\}$ be a set of degradable intervals. For all $i, 1 \leq i \leq n, \sigma_{i}$ is defined by $\left(r_{i}, q_{i}, d_{i}\right)$. For all $\sigma_{i} \in\left\{\sigma_{1}, \cdots, \sigma_{n}\right\}$, let $\sigma_{i}^{1}=\left[r_{i}, t_{i}^{1}\right)$ and $\sigma_{i}^{2}=\left[r_{i}, t_{i}^{2}\right)$ be two valid effective executions of $\sigma_{i}$ (i.e. $q_{i} \leq t_{i}^{1} \leq d_{i}$ and $q_{i} \leq t_{i}^{2} \leq d_{i}$ ). Let $E_{1} \subseteq\left\{\sigma_{i}^{1}: \sigma_{i} \in\right.$ $\left.\left\{\sigma_{1}, \ldots, \sigma_{n}\right\}\right\}$ and $E_{2} \subseteq\left\{\sigma_{i}^{2}: \sigma_{i} \in\left\{\sigma_{1}, \ldots, \sigma_{n}\right\}\right\}$. We define $E_{1} \uplus E_{2}$ the union ${ }^{+}$ of $E_{1}$ and $E_{2}$ as follows:

$-\sigma_{i}^{1} \in E_{1} \uplus E_{2}$ if and only if $\left(\sigma_{i}^{1} \in E_{1}\right.$ and $\left.\sigma_{i}^{2} \notin E_{2}\right)$ or $\left(\sigma_{i}^{1} \in E_{1}\right.$ and $\sigma_{i}^{2} \in$ $E_{2}$ and $\sigma_{i}^{1}$ covers $\sigma_{i}^{2}$ ).

$-\sigma_{i}^{2} \in E_{1} \uplus E_{2}$ if and only if $\left(\sigma_{i}^{2} \in E_{2}\right.$ and $\left.\sigma_{i}^{1} \notin E_{1}\right)$ or $\left(\sigma_{i}^{2} \in E_{2}\right.$ and $\sigma_{i}^{1} \in$ $E_{1}$ and $\sigma_{i}^{2}$ covers $\sigma_{i}^{1}$ ).

Note that the union ${ }^{+}$is commutative and it generalizes the usual definition of the union of two non-degradable intervals sets since in that case, $\sigma_{i}^{1}=\sigma_{i}^{2}$. Thus, for all $\sigma, E_{1}$ and $E_{2}$, if $\sigma \in E_{1} \uplus E_{2}$, then $\sigma \in E_{1} \cup E_{2}$.

As, by definition, the two effective executions of a same interval $\sigma$ defined by $(r, q, d)$ must start at the same release date $r$, the one with the smallest execution time is covered by the other.

Note that, to be completely rigorous, we should not define an interval $\sigma_{i}$ by $\left(r_{i}, q_{i}, d_{i}\right)$, but by $\left(r_{i}, q_{i}, d_{i}, i\right)$. Indeed, let us consider the following problematic example. Let $\sigma_{i}^{1}=\left[r_{i}, t_{i}^{1}\right)$ be an effective execution of $\sigma_{i}=\left(r_{i}, q_{i}, d_{i}\right)$ and $\sigma_{j}^{1}=\left[r_{j}, t_{j}^{1}\right)$ be an effective execution of $\sigma_{j}=\left(r_{j}, q_{j}, d_{j}\right)$, with $i \neq j$. If we consider the particular case where $r_{i}=r_{j}$ and $t_{i}^{1}=t_{j}^{1}$ (our model allows such a situation), then we have $\sigma_{i}^{1}=\left[r_{i}, t_{i}^{1}\right)=\left[r_{j}, t_{j}^{1}\right)=\sigma_{j}^{1}$. Of course, in this paper, we consider that the intervals are distinct (i.e. $\sigma_{i}^{1} \neq \sigma_{j}^{1}$ ). That is why we should define an interval $\sigma_{i}$ by $\left(r_{i}, q_{i}, d_{i}, i\right)$ and an effective execution $\sigma_{i}^{1}$ by $\left(\left[r_{i}, t_{i}^{1}\right), i\right)$. But, in order to simplify the notations, we write $\sigma_{i}=\left(r_{i}, q_{i}, d_{i}\right)$ instead of $\sigma_{i}=\left(r_{i}, q_{i}, d_{i}, i\right)$, and $\sigma_{i}^{1}=\left[r_{i}, t_{i}^{1}\right)$ instead of $\sigma_{i}^{1}=\left(\left[r_{i}, t_{i}^{1}\right), i\right)$.

The algorithm $A B^{k}$. The main idea is the following. $A B^{k}$ is running on $k$ identical machines (called real machines because it is on these machines that 
the effective schedule is built). It uses as subroutines $G O L$ and $L R$ (described in Section 1). For the ease of notation, we use $A$ for $G O L$ and $B$ for $L R$. For each new submitted interval $\sigma_{i}$, we simulate the execution of the algorithm $A^{r}$ (resp. $B^{k-r}$ ) on $r$ (resp. $k-r$ ) virtual machines, in order to control the size (resp. the weight) of the schedule. These two simulations (for the size and for the weight) are made on machines that we call virtual, because they are used only in order to determine the set (potentially empty) of intervals $A B^{k}$ has to interrupt and whether $\sigma_{i}$ has to be rejected or served by $A B^{k}$ (and in this last case, to decide in which degraded version $A B^{k}$ has to serve the new interval). Indeed, $A B^{k}$ serves $\sigma_{i}$ on a real machine if and only if $A^{r}$ or $B^{k-r}$ serves $\sigma_{i}$ (note that if both $A^{r}$ and $B^{k-r}$ serve it, $A B^{k}$ chooses the effective execution of $\sigma_{i}$ that covers the other).

In order to determine the schedule given by an algorithm after the interrupting and the scheduling stages, we introduce the following notation.

Notation 2 (Schedule returned by an algorithm on step $i$ ) For every on-line sequence $\sigma_{1}, \ldots, \sigma_{n}$, for every algorithm ALG and for every step of execution $i(1 \leq i \leq n)$ of $A L G$, let $\mathcal{O}_{i_{1}}(A L G)$ (resp. $\left.\mathcal{O}_{i_{2}}(A L G)\right)$ be the schedule returned $A L G$ after the interrupting (resp. scheduling) stage of step $i$.

Notation 3 (Set of intervals scheduled by $A B^{k}$ ) For every on-line sequence $\sigma_{1}, \ldots, \sigma_{n}$, for every step of execution $i(1 \leq i \leq n)$ of the algorithm $A B^{k}$, let $\mathcal{R}_{i_{1}}\left(A B^{k}\right)$ (resp. $\mathcal{R}_{i_{2}}\left(A B^{k}\right)$ ) be the set of intervals scheduled and not interrupted after the interrupting (resp. the scheduling) stage of step $i$ on the $k$ machines associated to $A B^{k}$, called real machines.

Notation 4 (Set of intervals scheduled by $A^{r}$ and $B^{k-r}$ ) For every online sequence $\sigma_{1}, \ldots, \sigma_{n}$, for every step of execution $i(1 \leq i \leq n)$ of the algorithm $A^{r}$ (resp. $\left.B^{k-r}\right)$, let $\mathcal{V}_{i_{1}}\left(A^{r}\right)$ (resp. $\left.\mathcal{V}_{i_{1}}\left(B^{k-r}\right)\right)$ be the set of intervals scheduled and not interrupted after the interrupting stage of step $i$ on the $r$ (resp $k-r)$ machines associated to $A^{r}$ (resp. $\left.B^{k-r}\right)$. Let $\mathcal{V}_{i_{2}}\left(A^{r}\right)\left(\right.$ resp. $\mathcal{V}_{i_{2}}\left(B^{k-r}\right)$ ) be the set of intervals scheduled and not interrupted after the scheduling stage of step $i$ on the $r$ (resp. $k-r)$ machines associated to $A^{r}$ (resp. $\left.B^{k-r}\right)$. The $r$ (resp $k-r)$ machines associated to $A^{r}$ (resp. $\left.B^{k-r}\right)$ are called virtual machines.

We give now a formal description of the algorithm $A B^{k}$.

Input: An on-line sequence of intervals $\sigma_{1}, \ldots, \sigma_{n}$ and $k$ identical machines.

Output: After each step $i(1 \leq i \leq n)$, a valid schedule $\mathcal{O}_{i_{2}}\left(A B^{k}\right)$ of $\sigma_{1}, \ldots, \sigma_{i}$ on the $k$ real machines.

Step 0: $\mathcal{V}_{0_{2}}\left(A^{r}\right)=\mathcal{V}_{0_{2}}\left(B^{k-r}\right)=\mathcal{R}_{0_{2}}\left(A B^{k}\right)=\emptyset$

Step i (date $\left.r_{i}\right)$ :

1. The interrupting stage of $A B^{k}$ :

(a) Execute the interrupting stage of $A^{r}$ (resp. $B^{k-r}$ ) on the $r$ (resp. $k-r$ ) virtual machines associated to $A^{r}$ (resp. $B^{k-r}$ ) by submitting the new interval $\sigma_{i}$ to $A^{r}$ (resp. $B^{k-r}$ ). Note that the set of intervals scheduled and not interrupted by $A^{r}\left(\operatorname{resp} . B^{k-r}\right)$ is now $\mathcal{V}_{i_{1}}\left(A^{r}\right)$ $\left(\operatorname{resp} . \mathcal{V}_{i_{1}}\left(B^{k-r}\right)\right)$. 
(b) Execute the interrupting stage of $A B^{k}$ on the $k$ real machines associated to $A B^{k}$ by interrupting the subset of intervals of $\mathcal{R}_{(i-1)_{2}}\left(A B^{k}\right)$ such that:

$$
\mathcal{R}_{i_{1}}\left(A B^{k}\right)=\mathcal{V}_{i_{1}}\left(A^{r}\right) \uplus \mathcal{V}_{i_{1}}\left(B^{k-r}\right)
$$

2. The scheduling stage of $A B^{k}$ :

(a) Execute the scheduling stage of $A^{r}$ (resp. $B^{k-r}$ ) on the $r$ (resp. $k-r$ ) virtual machines associated to $A^{r}$ (resp. $B^{k-r}$ ) by serving or rejecting the new interval $\sigma_{i}$.

(b) Execute the scheduling stage of $A B^{k}$ on the $k$ real machines associated to $A B^{k}$ by switching to the appropriate case:

i. If $A^{r}$ and $B^{k-r}$ reject $\sigma_{i}$, then $A B^{k}$ does not schedule $\sigma_{i}$. Thus, we have: $\quad \mathcal{R}_{i_{2}}\left(A B^{k}\right)=\mathcal{R}_{i_{1}}\left(A B^{k}\right)$

ii. If $A^{r}$ serves $\sigma_{i}$ (with effective execution $\sigma_{i}^{A}$ ) and $B^{k-r}$ rejects $\sigma_{i}$ then $A B^{k}$ serves $\sigma_{i}^{A}$ on any free machine and we have:

$$
\mathcal{R}_{i_{2}}\left(A B^{k}\right)=\mathcal{R}_{i_{1}}\left(A B^{k}\right) \cup\left\{\sigma_{i}^{A}\right\}
$$

iii. If $A^{r}$ rejects $\sigma_{i}$ and $B^{k-r}$ serves $\sigma_{i}$ (with effective execution $\sigma_{i}^{B}$ ) then $A B^{k}$ serves $\sigma_{i}^{B}$ on any free machine and we have:

$$
\mathcal{R}_{i_{2}}\left(A B^{k}\right)=\mathcal{R}_{i_{1}}\left(A B^{k}\right) \cup\left\{\sigma_{i}^{B}\right\}
$$

iv. If $A^{r}$ and $B^{k-r}$ serve $\sigma_{i}$ one with effective execution $\sigma_{i}^{A}$ and the other with effective execution $\sigma_{i}^{B}$ then $A B^{k}$ serves the effective execution that covers the other on any free machine. If $\sigma_{i}^{B}$ covers $\sigma_{i}^{A}$ then we have:

else we have:

$$
\mathcal{R}_{i_{2}}\left(A B^{k}\right)=\mathcal{R}_{i_{1}}\left(A B^{k}\right) \cup\left\{\sigma_{i}^{B}\right\}
$$

$$
\mathcal{R}_{i_{2}}\left(A B^{k}\right)=\mathcal{R}_{i_{1}}\left(A B^{k}\right) \cup\left\{\sigma_{i}^{A}\right\}
$$

Intervals scheduled by the algorithm $A B^{k}$. We first present Lemma 3 which states that the algorithm $A B^{k}$ schedules the same intervals as the union ${ }^{+}$of the intervals scheduled by $A^{r}$ and the intervals scheduled by $B^{k-r}$.

Lemma 3 For each step $i$ of execution of the algorithm $A B^{k}$, the schedule $\mathcal{O}_{i_{2}}\left(A B^{k}\right)$ is valid and $\mathcal{R}_{i_{2}}\left(A B^{k}\right)=\mathcal{V}_{i_{2}}\left(A^{r}\right) \uplus \mathcal{V}_{i_{2}}\left(B^{k-r}\right)$.

Proof. We prove Lemma 3 by induction on the steps of execution $i$ of $A B^{k}$.

The basic case (step 0): By definition of $A B^{k}$, we have $\mathcal{V}_{0_{2}}\left(A^{r}\right)=\mathcal{V}_{0_{2}}\left(B^{k-r}\right)=$ $\mathcal{R}_{0_{2}}\left(A B^{k}\right)=\emptyset$. Thus, $\mathcal{O}_{i_{2}}\left(A B^{k}\right)$ is valid and we have $\mathcal{R}_{i_{2}}\left(A B^{k}\right)=\mathcal{V}_{i_{2}}\left(A^{r}\right) \uplus$ $\mathcal{V}_{i_{2}}\left(B^{k-r}\right)$. The basic case is checked.

The main case (step i): Let us assume that $\mathcal{O}_{(i-1)_{2}}\left(A B^{k}\right)$ is valid and that $\mathcal{R}_{(i-1)_{2}}\left(A B^{k}\right)=\mathcal{V}_{(i-1)_{2}}\left(A^{r}\right) \uplus \mathcal{V}_{(i-1)_{2}}\left(B^{k-r}\right)$ (by the assumption of the induction).

1. The interrupting stage: We first need to prove that $\mathcal{R}_{i_{1}}\left(A B^{k}\right)=\mathcal{V}_{i_{1}}\left(A^{r}\right) \uplus$ $\mathcal{V}_{i_{1}}\left(B^{k-r}\right)$ and that $\mathcal{O}_{i_{1}}\left(A B^{k}\right)$ is valid.

(a) By definition, $A B^{k}$ interrupts the subset of intervals of $\mathcal{R}_{(i-1)_{2}}\left(A B^{k}\right)$ such that:

$$
\mathcal{R}_{i_{1}}\left(A B^{k}\right)=\mathcal{V}_{i_{1}}\left(A^{r}\right) \uplus \mathcal{V}_{i_{1}}\left(B^{k-r}\right) \quad \text { (UNION) }
$$


We have to show that there is always a subset of intervals of $\mathcal{R}_{(i-1)_{2}}\left(A B^{k}\right)$ that can be removed such that the above equality is possible.

Since $\mathcal{V}_{i_{1}}\left(A^{r}\right) \subseteq \mathcal{V}_{(i-1)_{2}}\left(A^{r}\right), \mathcal{V}_{i_{1}}\left(B^{k-r}\right) \subseteq \mathcal{V}_{(i-1)_{2}}\left(B^{k-r}\right)$, and $\mathcal{R}_{(i-1)_{2}}\left(A B^{k}\right)=\mathcal{V}_{(i-1)_{2}}\left(A^{r}\right) \uplus \mathcal{V}_{(i-1)_{2}}\left(B^{k-r}\right)$ (by the assumption of the induction), we have $\mathcal{V}_{i_{1}}\left(A^{r}\right) \uplus \mathcal{V}_{i_{1}}\left(B^{k-r}\right) \subseteq \mathcal{R}_{(i-1)_{2}}\left(A B^{k}\right)$.

(b) By definition, $A B^{k}$ interrupts only intervals scheduled in $\mathcal{O}_{(i-1)_{2}}\left(A B^{k}\right)$, and by assumption of induction, $\mathcal{O}_{(i-1)_{2}}\left(A B^{k}\right)$ is valid. Thus, there cannot be intervals scheduled at the same time or more than once in $\mathcal{O}_{i_{1}}\left(A B^{k}\right)$. This means that $\mathcal{O}_{i_{1}}\left(A B^{k}\right)$ is valid.

(VALID)

2. The scheduling stage: We now prove that $\mathcal{R}_{i_{2}}\left(A B^{k}\right)=\mathcal{V}_{i_{2}}\left(A^{r}\right) \uplus \mathcal{V}_{i_{2}}\left(B^{k-r}\right)$ and that $\mathcal{O}_{i_{2}}\left(A B^{k}\right)$ is valid. By definition of $A B^{k}$, several cases may happen: (a) If $A^{r}$ and $B^{k-r}$ reject $\sigma_{i}$, then $A B^{k}$ does not schedule $\sigma_{i}$ and we have:

i.

$$
\begin{aligned}
\mathcal{R}_{i_{2}}\left(A B^{k}\right)= & \mathcal{R}_{i_{1}}\left(A B^{k}\right)=\mathcal{V}_{i_{1}}\left(A^{r}\right) \uplus \mathcal{V}_{i_{1}}\left(B^{k-r}\right) \\
& \left(\text { by the definition of } A B^{k} \text { and by }(\mathrm{UNION})\right) \\
= & \mathcal{V}_{i_{2}}\left(A^{r}\right) \uplus \mathcal{V}_{i_{2}}\left(B^{k-r}\right) \\
& \left(\text { since } A^{r} \text { and } B^{k-r} \text { reject } \sigma_{i},\right. \text { we have } \\
& \left.\mathcal{V}_{i_{1}}\left(A^{r}\right)=\mathcal{V}_{i_{2}}\left(A^{r}\right) \text { and } \mathcal{V}_{i_{1}}\left(B^{k-r}\right)=\mathcal{V}_{i_{2}}\left(B^{k-r}\right)\right)
\end{aligned}
$$

ii. $\mathcal{O}_{i_{2}}\left(A B^{k}\right)=\mathcal{O}_{i_{1}}\left(A B^{k}\right)$. Thus $\mathcal{O}_{i_{2}}\left(A B^{k}\right)$ is valid (because by (VALID), $\mathcal{O}_{i_{1}}\left(A B^{k}\right)$ is valid).

(b) If $A^{r}$ serves $\sigma_{i}$ (with effective execution $\sigma_{i}^{A}$ ) and $B^{k-r}$ rejects $\sigma_{i}$, then $A B^{k}$ schedules $\sigma_{i}^{A}$ on any free real machine at time $r_{i}$ and we have:

i.

$$
\begin{aligned}
\mathcal{R}_{i_{2}}\left(A B^{k}\right)= & \mathcal{R}_{i_{1}}\left(A B^{k}\right) \cup\left\{\sigma_{i}^{A}\right\}=\left(\mathcal{V}_{i_{1}}\left(A^{r}\right) \uplus \mathcal{V}_{i_{1}}\left(B^{k-r}\right)\right) \cup\left\{\sigma_{i}^{A}\right\} \\
& \left(\text { by the definition of } A B^{k} \text { and by }(\text { UNION })\right) \\
= & \mathcal{V}_{i_{2}}\left(A^{r}\right) \uplus \mathcal{V}_{i_{2}}\left(B^{k-r}\right) \\
& \left(\text { union and union }{ }^{+} \text {commute and since } A^{r} \text { serves } \sigma_{i}\right. \\
& \text { and } B^{k-r} \text { rejects } \sigma_{i}, \text { we have } \mathcal{V}_{i_{2}}\left(A^{r}\right)=\mathcal{V}_{i_{1}}\left(A^{r}\right) \cup\left\{\sigma_{i}^{A}\right\} \\
& \text { and } \left.\mathcal{V}_{i_{2}}\left(B^{k-r}\right)=\mathcal{V}_{i_{1}}\left(B^{k-r}\right)\right)
\end{aligned}
$$

ii. Since $\mathcal{O}_{i_{1}}\left(A B^{k}\right)$ is a valid schedule (by (VALID)) and $\mathcal{O}_{i_{2}}\left(A B^{k}\right)$ is built by $A B^{k}$ by adding $\sigma_{i}$ to $\mathcal{O}_{i_{1}}\left(A B^{k}\right)$ only once, the only reason for which $\mathcal{O}_{i_{2}}\left(A B^{k}\right)$ could not be valid would be because $\sigma_{i}$ is scheduled by $A B^{k}$ at time $r_{i}$ whereas there is no free machine at time $r_{i}$, i.e. because there are at least $k+1$ intervals of $\mathcal{R}_{i_{2}}\left(A B^{k}\right)$ scheduled at time $r_{i}$ by $A B^{k}$. Let us prove that this is impossible. Indeed, since $A^{r}$ and $B^{k-r}$ build at each time valid schedules, there are at most $r+k-r=k$ intervals of $\mathcal{V}_{i_{2}}\left(A^{r}\right) \uplus \mathcal{V}_{i_{2}}\left(B^{k-r}\right)$ scheduled at time $r_{i}$ by $A^{r}$ and $B^{k-r}$, and thus, there are at most $k$ intervals of $\mathcal{R}_{i_{2}}\left(A B^{k}\right)$ scheduled at time $r_{i}$ by $A B^{k}$ (because we just proved above that $\left.\mathcal{R}_{i_{2}}\left(A B^{k}\right)=\mathcal{V}_{i_{2}}\left(A^{r}\right) \uplus \mathcal{V}_{i_{2}}\left(B^{k-r}\right)\right)$. Thus, $\mathcal{O}_{i_{2}}\left(A B^{k}\right)$ is a valid schedule.

(c) If $A^{r}$ rejects $\sigma_{i}$ and $B^{k-r}$ serves $\sigma_{i}$ (with effective execution $\sigma_{i}^{B}$ ), then $A B^{k}$ schedules $\sigma_{i}^{B}$ on any free real machine at time $r_{i}$. 
i. We prove that $\mathcal{R}_{i_{2}}\left(A B^{k}\right)=\mathcal{V}_{i_{2}}\left(A^{r}\right) \uplus \mathcal{V}_{i_{2}}\left(B^{k-r}\right)$ in the same way that we prove it in $2(\mathrm{~b}) \mathrm{i}$, except that we replace $\sigma_{i}^{A}$ by $\sigma_{i}^{B}$.

ii. We prove that $\mathcal{O}_{i_{2}}\left(A B^{k}\right)$ is valid in the same way as in 2(b)ii.

(d) If $A^{r}$ and $B^{k-r}$ serve $\sigma_{i}$ one with effective execution $\sigma_{i}^{A}$ and the other with effective execution $\sigma_{i}^{B}$. Let $\sigma_{i}^{S}$ (resp. $\sigma_{i}^{L}$ ) be the shortest (resp. longest) effective execution of $\sigma_{i}$. Without loss of generality, we suppose that $A^{r}$ schedules $\sigma_{i}^{S}$ and $B^{k-r}$ schedules $\sigma_{i}^{L}$. By definition of the algorithm, $A B^{k}$ schedules $\sigma_{i}^{L}$, and we have:

i.

$$
\begin{aligned}
\mathcal{R}_{i_{2}}\left(A B^{k}\right)= & \mathcal{R}_{i_{1}}\left(A B^{k}\right) \cup\left\{\sigma_{i}^{L}\right\}=\left(\mathcal{V}_{i_{1}}\left(A^{r}\right) \uplus \mathcal{V}_{i_{1}}\left(B^{k-r}\right)\right) \cup\left\{\sigma_{i}^{L}\right\} \\
& \left(\text { by definition of } A B^{k} \text { and by }(\text { UnION })\right) \\
= & \left(\mathcal{V}_{i_{1}}\left(A^{r}\right) \uplus \mathcal{V}_{i_{1}}\left(B^{k-r}\right)\right) \cup\left(\left\{\sigma_{i}^{L}\right\} \uplus\left\{\sigma_{i}^{S}\right\}\right) \\
& \left(\text { because, by definition of union },\left\{\sigma_{i}^{L}\right\}=\left\{\sigma_{i}^{L}\right\} \uplus\left\{\sigma_{i}^{S}\right\}\right) \\
= & \mathcal{V}_{i_{1}}\left(A^{r}\right) \uplus \mathcal{V}_{i_{1}}\left(B^{k-r}\right) \uplus\left\{\sigma_{i}^{L}\right\} \uplus\left\{\sigma_{i}^{S}\right\} \\
& \left(\text { because }\left(\mathcal{V}_{i_{1}}\left(A^{r}\right) \uplus \mathcal{V}_{i_{1}}\left(B^{k-r}\right)\right) \cap\left(\left\{\sigma_{i}^{L}\right\} \uplus\left\{\sigma_{i}^{S}\right\}\right)=\emptyset\right) \\
= & \left(\mathcal{V}_{i_{1}}\left(A^{r}\right) \cup\left\{\sigma_{i}^{S}\right\}\right) \uplus\left(\mathcal{V}_{i_{1}}\left(B^{k-r}\right) \cup\left\{\sigma_{i}^{L}\right\}\right) \\
& \left(\text { because the union }{ }^{+} \text {is commutative and since } \sigma_{i}^{S} \notin\right. \\
& \left.\mathcal{V}_{i_{1}}\left(A^{r}\right), \text { we have } \mathcal{V}_{i_{1}}\left(A^{r}\right) \uplus\left\{\sigma_{i}^{S}\right\}=\mathcal{V}_{i_{1}}\left(A^{r}\right) \cup\left\{\sigma_{i}^{S}\right\}\right) \\
= & \mathcal{V}_{i_{2}}\left(A^{r}\right) \uplus \mathcal{V}_{i_{2}}\left(B^{k-r}\right) \\
& \left(\text { because since } A^{r} \text { and } B^{k-r} \text { serve } \sigma_{i}, \text { we have } \mathcal{V}_{i_{2}}\left(A^{r}\right)\right. \\
& \left.=\mathcal{V}_{i_{1}}\left(A^{r}\right) \cup\left\{\sigma_{i}^{S}\right\} \text { and } \mathcal{V}_{i_{2}}\left(B^{k-r}\right)=\mathcal{V}_{i_{1}}\left(B^{k-r}\right) \cup\left\{\sigma_{i}^{L}\right\}\right)
\end{aligned}
$$

ii. We prove that $\mathcal{O}_{i_{2}}\left(A B^{k}\right)$ is valid in the same way as in $2(\mathrm{~b})$ ii.

Corollary 2 Let $A^{r}=G O L^{r}$ and $B^{k-r}=L R^{k-r}$. Let $N\left(\mathcal{V}_{i_{2}}\left(G O L^{r}\right)\right)=$ $\left|\mathcal{V}_{i_{2}}\left(G O L^{r}\right)\right|$ and $W\left(\mathcal{V}_{i_{2}}\left(L R^{k-r}\right)\right)$ be the sum of the weight of the intervals of $\mathcal{V}_{i_{2}}\left(L R^{k-r}\right)$. For every input sequence $\sigma_{1}, \ldots, \sigma_{n}$ and for every step $i(1 \leq i \leq n)$ of the algorithm $A B^{k}$, we have:

$$
N\left(\mathcal{V}_{i_{2}}\left(G O L^{r}\right)\right) \leq N\left(\mathcal{R}_{i_{2}}\left(A B^{k}\right)\right) \quad \text { and } \quad W\left(\mathcal{V}_{i_{2}}\left(L R^{k-r}\right)\right) \leq W\left(\mathcal{R}_{i_{2}}\left(A B^{k}\right)\right)
$$

Proof. By Lemma 3, for every step $i$ of the algorithm $A B^{k}$, we have $\mathcal{R}_{i_{2}}\left(A B^{k}\right)=$ $\mathcal{V}_{i_{2}}\left(A^{r}\right) \uplus \mathcal{V}_{i_{2}}\left(B^{k-r}\right)=\mathcal{V}_{i_{2}}\left(G O L^{r}\right) \uplus \mathcal{V}_{i_{2}}\left(L R^{k-r}\right)$, thus, by definition of union ${ }^{+}$, Corollary 2 is checked.

Theorem 1. For all $k \geq 4$, for all $r, 1 \leq r \leq k-3$, the algorithm $A B^{k}$ applied with $G O L^{r}$ and $L R^{k-r}$ is $\left(\frac{k}{r}, \frac{4 k}{k-r-2}\right)$-competitive for the Size and Proportional weights metrics.

Proof. Let $\sigma_{1}, \cdots, \sigma_{n}$ be any on-line sequence of intervals and let $O_{x}^{N *}$ (resp. $O_{x}^{W *}$ ) be an optimal schedule of $\left\{\sigma_{1}, \cdots, \sigma_{n}\right\}$ for the size $N$ (resp. for the proportional weight $W$ ) on $x \leq k$ machines. Let $O_{r}^{G O L}$ (resp. $O_{k-r}^{L R}$ ) be the schedule returned by $G O L^{r}$ (resp. $L R^{k-r}$ ) on the on-line sequence $\sigma_{1}, \cdots, \sigma_{n}$ on $r \leq k-3$ (resp. $k-r \geq 3$ ) machines. Since, by Lemma $1, G O L^{r}$ is 1-competitive (resp. by Corollary $1, L R^{k-r}$ is $\left(\frac{4}{1-\frac{2}{k-r}}\right)$-competitive), we have: 


$$
N\left(O_{r}^{N *}\right) \leq N\left(O_{r}^{G O L}\right) \quad\left(\text { resp. } W\left(O_{k-r}^{W *}\right) \leq\left(\frac{4}{1-\frac{2}{k-r}}\right) W\left(O_{k-r}^{L R}\right)\right)
$$

Let $O^{\prime N}$ (resp. $O^{\prime W}$ ) be the $r$ (resp. $k-r$ ) machine sub-schedule of $O_{k}^{N *}$ (resp. $O_{k}^{W *}$ ) executing all the intervals appearing on the $r$ (resp. $k-r$ ) machines of $O_{k}^{N *}\left(\right.$ resp. $\left.O_{k}^{W *}\right)$ generating the largest size (resp. weight). Since $O^{\prime N}$ (resp. $\left.O^{\prime W}\right)$ is a $r($ resp. $k-r)$ machine schedule, we have $N\left(O^{\prime N}\right) \leq N\left(O_{r}^{N *}\right)$ (resp. $W\left(O^{\prime W}\right) \leq W\left(O_{k-r}^{W *}\right)$ ), otherwise, $O_{r}^{N *}$ (resp. $O_{k-r}^{W *}$ ) would not be an optimal schedule for the size (resp. weight). Combined with (1), we obtain:

$$
\begin{gathered}
N\left(O^{\prime N}\right) \leq N\left(O_{r}^{N *}\right) \leq N\left(O_{r}^{G O L}\right) \\
\left(\text { resp. } W\left(O^{\prime W}\right) \leq W\left(O_{k-r}^{W *}\right) \leq\left(\frac{4}{1-\frac{2}{k-r}}\right) W\left(O_{k-r}^{L R}\right)\right)
\end{gathered}
$$

Since $O^{\prime N}$ (resp. $O^{\prime W}$ ) is the $r$ machine sub-schedule of $O_{k}^{N *}$ (resp. $O_{k}^{W *}$ ) generating the largest size (resp. weight), the average size (resp. weight) per machine in $O^{\prime N}$ (resp. $O^{\prime W}$ ) is larger than the average size (resp. weight) per machine in $O_{k}^{N *}$ (resp. $\left.O_{k}^{W *}\right)$. Thus, we have $\frac{N\left(O_{k}^{N *}\right)}{k} \leq \frac{N\left(O^{\prime N}\right)}{r} \Rightarrow N\left(O_{k}^{N *}\right) \leq \frac{k}{r} N\left(O^{\prime N}\right)$ (resp. $\left.\frac{W\left(O_{k}^{W *}\right)}{k} \leq \frac{W\left(O^{\prime W}\right)}{k-r} \Rightarrow W\left(O_{k}^{W *}\right) \leq \frac{k}{k-r} W\left(O^{\prime W}\right)\right)$. Combined with (2), we obtain:

$$
N\left(O_{k}^{N *}\right) \leq \frac{k}{r} N\left(O_{r}^{G O L}\right) \quad\left(\operatorname{resp} . W\left(O_{k}^{W *}\right) \leq \frac{4 k}{k-r-2} W\left(O_{k-r}^{L R}\right)\right)
$$

As $N\left(O_{r}^{G O L}\right)=N\left(\mathcal{V}_{i_{2}}\left(G O L^{r}\right)\right)\left(\right.$ resp. $\left.W\left(O_{k-r}^{L R}\right)=W\left(\mathcal{V}_{i_{2}}\left(L R^{k-r}\right)\right)\right)$, by applying Corollary 2 on (3), we obtain:

$$
\begin{aligned}
N\left(O_{k}^{N *}\right) & \leq \frac{k}{r} N\left(\mathcal{V}_{i_{2}}\left(G O L^{r}\right)\right) \leq \frac{k}{r} N\left(\mathcal{R}_{i_{2}}\left(A B^{k}\right)\right) \\
\left(\operatorname{resp.} W\left(O_{k}^{W *}\right)\right. & \left.\leq \frac{k}{r} W\left(\mathcal{V}_{i_{2}}\left(L R^{k-r}\right)\right) \leq \frac{4 k}{k-r-2} W\left(\mathcal{R}_{i_{2}}\left(A B^{k}\right)\right)\right)
\end{aligned}
$$

This means that $A B^{k}$ is $\left(\frac{k}{r}, \frac{4 k}{k-r-2}\right)$-competitive.

Example. If $r=\frac{k}{2}$ (if $\frac{k}{2} \geq 3$ and $k$ even), $A B^{k}$ is $\left(2, \frac{8}{1-\frac{4}{k}}\right)$-competitive.

\section{References}

1. E. Arkin And B. Silverberg, Scheduling jobs with fixed start and end times, Discrete Applied Mathematics, 18 (1987), pp. 1-8.

2. F. BAille, E. BAMPIS, AND C. LAFOREST, Maximization of the size and the weight of schedules of degradable intervals, in proceedings of COCOON'04, K.-Y. Chwa and I. Munro, eds., LNCS No. 3106, Springer-Verlag, 2004, pp. 219-228.

3 . — A note on bicriteria schedules with optimal approximation ratios, Parallel Processing Letters, 14 (2004), pp. 315-323.

4. A. Bar-Noy, R. Canetti, S. Kutten, Y. Mansour, and B. Schieber, Bandwidth allocation with preemption, SIAM J. Comput., 28 (1999), pp. 1806-1828.

5. A. Borodin AND R. El-YANIV, Online computation and competitive analysis, Cambridge University press, 1998.

6. M. C. Carlisle and E. L. Lloyd, On the $k$-coloring of intervals, Discrete Applied Mathemetics, 59 (1995), pp. 225-235.

7. U. FAigle And M. NAWIJn, Note on scheduling intervals on-line, Discrete Applied Mathematics, 58 (1995), pp. 13-17.

8. G. J. Woeginger, On-line scheduling of jobs with fixed start and end times, Theor. Comput. Sci., 130 (1994), pp. 5-16. 